\title{
The Fostering Resilience through Art in Medical Education (FRAME) workshop: a partnership with the Philadelphia Museum of Art
}

This article was published in the following Dove Press journal:

Advances in Medical Education and Practice

\author{
Andrew R Orr' \\ Nazanin Moghbeli ${ }^{1}$ \\ Amanda Swain ${ }^{2}$ \\ Barbara Bassett ${ }^{3}$ \\ Suzannah Niepold ${ }^{3}$ \\ Adam Rizzo ${ }^{3}$ \\ Horace M DeLisser ${ }^{4}$ \\ 'Department of Medicine, Perelman \\ School of Medicine, University of \\ Pennsylvania, Philadelphia, PA, USA; \\ ${ }^{2}$ Department of Family Medicine and \\ Community Health, Perelman School of \\ Medicine, University of Pennsylvania, \\ Philadelphia, PA, USA; ${ }^{3}$ Division of \\ Education, Philadelphia Museum of Art, \\ Philadelphia, USA; ${ }^{4}$ Academic Programs \\ Office, Perelman School of Medicine, \\ University of Pennsylvania, Philadelphia, \\ PA, USA
}

Background: Provider burnout remains a serious problem facing medical training programs and has been shown to affect more than half of internal medicine residents. In addition to broader efforts to revamp a health care system that contributes to this epidemic, exposure to the medical humanities offers potential to promote engagement, resilience, and restoration of meaning in residents' daily lives.

Objective: We aim to create a reproducible, evidence-based workshop utilizing artful thinking routines to prepare trainees to combat burnout with reflection, perspective-taking, and community-building.

Methods: A single, 4-hour workshop for senior internal medicine residents, centered on visual artistic analysis, was offered in June 2017 at the Philadelphia Museum of Art. Pre- and post-workshop burnout metrics and survey evaluation data were analyzed using a mixedmethods approach.

Results: Workshop participation was offered to 29 internal medicine residents, of whom 17 $(59 \%)$ participated. All survey respondents $(n=13)$ rated the workshop as excellent and would recommend it to colleagues. Moderate decreases in the observed frequencies of both high emotional exhaustion scores $(64.7 \%$ before the workshop to $55.5 \%$ following the workshop) and high depersonalization scores $(70.6 \%$ before the workshop to $55.5 \%$ following the workshop) were observed.

Conclusions: While results are preliminary in nature, the workshop was received favorably and demonstrated modest decreases in emotional exhaustion and depersonalization. We are encouraged to explore and repeat this workshop with modifications to identify its optimal position in the broader landscape of emerging wellness curricula.

Keywords: burnout, medical humanities, resilience, visual art, medical education

\section{Introduction}

Provider burnout remains a serious problem facing medical training programs. ${ }^{1,2}$ Characterized as a constellation of emotional exhaustion, depersonalization, and low personal accomplishment, the burnout syndrome begins as early as undergraduate medical education and subsequently increases among practicing physicians. ${ }^{3}$ Among US Internal Medicine residents, the target population of this study, the reported burnout rate is more than $50 \% .{ }^{4}$ While previous studies have clearly identified drivers of burnout, ${ }^{5,6}$ few interventions to date have been shown to significantly decrease it. ${ }^{7}$

Current, evidence-based interventions include mindfulness training and relaxation techniques. ${ }^{8}$ In similar fashion, exposure to the medical humanities seems to
Correspondence: Andrew R Orr Department of Medicine, Perelman School of Medicine, University of Pennsylvania, 3400 Spruce Street, 5 Maloney, Philadelphia, PA 19104, USA Tel + I 2156623797

Email andrew.orr@pennmedicine.upenn. edu 
offer an additional avenue to decrease the burnout burden. However, while humanistic interventions can increase empathy, their methods remain unstandardized and their benefits in burnout prevention have yet to be sufficiently investigated. ${ }^{9-11}$

In an effort to standardize the current "menu" of individual burnout interventions, we aim to create a reproducible workshop focused on visual arts. ${ }^{12-16}$ The Fostering Resilience through Art in Medical Education (FRAME) workshop utilizes Artful Thinking Routines established by Harvard Project Zero to model introductory artistic analysis and positive feedback. ${ }^{17}$ These routines are similar to, yet distinct from, previously validated Visual Thinking Strategies. ${ }^{18-22}$ The workshop combines these elements with the successful "Personal Responses Tour" format from Gaufberg and Williams. ${ }^{9}$ Through evidence-based standardization, we hope to maximize the potential of studying the visual arts' ability to enhance community-building and mitigate burnout in Internal Medicine residents.

\section{Methods}

\section{Study population}

Participation in the June 2017 workshop was offered to one cohort of twenty-nine Internal Medicine residents on ambulatory or elective rotations at a single academic institution. Study participation was voluntary, with participating residents exempt from clinic responsibilities during the session.

\section{Workshop design}

The FRAME workshop occurred at the Philadelphia Museum of Art (PMA). Individual exercises were designed and led by two PMA instructors. Workshop itinerary included the following four activities in succession: "Elaboration Game"17 (45 minutes), "Describe and Draw" (45 minutes), "Step Inside"17 (45 minutes), and the Personal Responses Tour" (75 minutes). See Supplementary material for further descriptions of each activity.

The seventeen residents remained together for the first three activities before being split into two groups for the Personal Responses Tour. Similarly, the four Internal Medicine faculty facilitators remained with the large group and then were divided equally between groups in the concluding activity. As each of the first three activities ran slightly longer than anticipated, the second half of the Personal Responses Tour was cut short by twenty minutes in order to accommodate the museum's allotted 4-hour time window.
Course objectives were listed as follows:

(i) Increase participant awareness of the importance of being thoughtful and flexible in thinking.

(ii) Provide an opportunity to practice relational communication skills.

(iii) Provide an opportunity for participants to reflect on an aspect of work that they find difficult, stressful, or overwhelming.

(iv) Provide an opportunity for participants to reflect on an aspect of work that they find important, meaningful, or satisfying.

\section{Course evaluation and analysis}

Immediately following the workshop, participants completed a nineteen-question mixed methods survey instrument to assess resident perception of the workshop.

\section{Burnout assessment}

We chose to use an abbreviated version of the validated Maslach burnout inventory (MBI) for analysis. ${ }^{23-25}$

One week prior to the workshop, the abbreviated MBI was administered to the FRAME cohort. The inventory was also sent to the remainder of the residency program's PGY-2 or PGY-3 residents at this time to confirm that there were no significant baseline differences in the study group. The same instrument was then sent again to the intervention group three months after the FRAME workshop as follow-up to assess for a sustainable reduction in burnout metrics.

The inventory questions were graded on a 7-point scale ranging from 0 to 6 , with higher scores indicating increased frequency of symptoms (never $=0$, a few times a year $=1$, once a month or less $=2$, a few times a month $=3$, once a week $=4$, a few times a week $=5$, every day $=6$ ) in each burnout category. As such, higher scores indicated greater depersonalization and emotional exhaustion but also greater personal accomplishment. Effect sizes were used to assess variation. Effect sizes of $0.25-0.30$ were characterized as small, $0.40-0.80$ as moderate, and above 0.80 as large (indicating a large difference between the two groups in question).

Finally, using the method validated by West et al, responses to the two, single questions validated as predictors of performance on the full MBI were also analyzed. 24 "I feel emotionally drained from my work" was used as a surrogate for "I feel burned out from my work" to represent emotional exhaustion. "I have become more callous toward people since I took this job" was used directly to represent overall depersonalization. We adopted the same metric for assigning 
a designation of "burnout" as in that study, with a symptom frequency of "at least weekly" indicating burnout. These symptom frequencies correlate with the most commonly used cutpoints for designating burnout via the full MBI in the literature: a score of 27 or greater for emotional exhaustion and/or a score of 10 or greater for depersonalization. ${ }^{26}$

Statistics were calculated using SPSS version 24.

The University of Pennsylvania Institution Review Board (IRB) exempted the study from IRB approval due to the absence of risk to subjects. Workshop participation was voluntary.

\section{Results}

\section{Baseline characteristics}

Of the twenty-nine residents to whom participation was offered, twenty residents $(69 \%)$ volunteered for the workshop and seventeen (59\%; 10 male, 7 female; 6 PGY-2, 10 PGY-3, and 1 PGY-4 combined medicine/pediatrics) participated.

The aggregate baseline scores in each burnout category of the FRAME group vs the control group can be found in Table 1 . The two groups demonstrated only a small difference in baseline depersonalization responses, with slightly higher depersonalization among the pre-intervention cohort.

\section{Burnout assessment}

Pre- and post-workshop abbreviated Maslach burnout inventory scores grouped by category are represented in Table 2. Nine of seventeen (53\%) workshop participants completed the post-workshop inventory. Moderate decreases in depersonalization and emotional exhaustion were observed among survey respondents, as well as a small increase in personal accomplishment.

Table I Mean baseline burnout scores and effect sizes

\begin{tabular}{|l|c|c|c|}
\hline & $\begin{array}{l}\text { Control group } \\
(\boldsymbol{n}=\mathbf{6 7})\end{array}$ & Pre-intervention (FRAME) group $(\boldsymbol{n}=\mathbf{I})$ & Effect size \\
\hline Depersonalization & $2.98 \pm 1.82$ & $3.49 \pm 1.80$ & 0.28 \\
Emotional exhaustion & $3.78 \pm 1.54$ & $3.75 \pm 1.39$ & -0.02 \\
Personal accomplishment & $4.99 \pm 0.87$ & $4.77 \pm 1.00$ & -0.24 \\
\hline
\end{tabular}

Abbreviation: FRAME, Fostering Resilience through Art in Medical Education.

Table 2 Mean burnout scores before and after the FRAME workshop

\begin{tabular}{|l|c|l|l|}
\hline & Pre-workshop group $(\boldsymbol{n}=\mathbf{1 7})$ & $\begin{array}{l}\text { Post-workshop group at 3 months } \\
(\boldsymbol{n}=\mathbf{9})\end{array}$ & Effect size \\
\hline Depersonalization & $3.49 \pm 1.80$ & $2.70 \pm 1.94$ & -0.41 \\
Emotional exhaustion & $3.75 \pm 1.39$ & $3.00 \pm 1.72$ & -0.47 \\
Personal accomplishment & $4.77 \pm 1.00$ & $5.15 \pm 0.92$ & 0.38 \\
\hline
\end{tabular}

Abbreviation: FRAME, Fostering Resilience through Art in Medical Education.

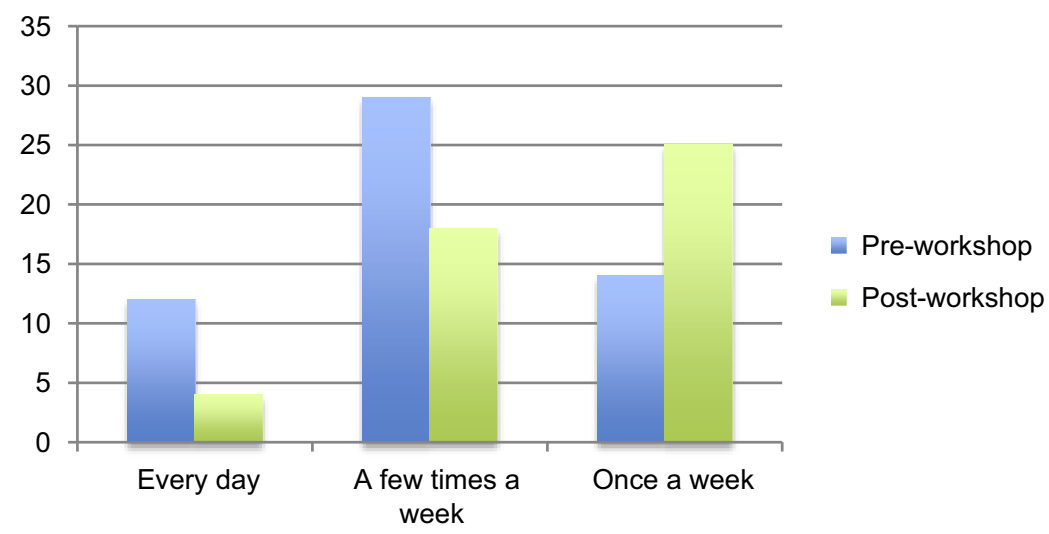

Figure I Pre- and post-workshop frequency of experiencing emotional exhaustion symptoms. 
The shift in reported frequency of symptoms in the aggregate emotional exhaustion category can be seen in Figure 1. Frequency of daily emotional exhaustion symptoms decreased from twelve percent to five percent after the intervention. Frequency of experiencing emotional exhaustion symptoms a few times a week decreased from twenty-nine to eighteen percent. Conversely, frequency of experiencing these symptoms only once a week increased from fourteen to twenty-five percent.

The shift in reported frequency of symptoms in the aggregate depersonalization category can be seen in Figure 2. Frequency of daily depersonalization symptoms decreased from sixteen percent to four percent after the workshop. The frequency of experiencing depersonalization symptoms a few times a week decreased from twenty-five to nineteen percent. Finally, the frequency of experiencing these symptoms only once a week increased from twelve to twenty-two percent.

Using the single item, validated approach to the MBI, the percentage of respondents demonstrating burnout by emotional exhaustion criterion decreased from $64.7 \%$ to $55.5 \%$ after the workshop. Similarly, the rate of burnout by depersonalization criterion decreased from $70.6 \%$ to $55.5 \%$.

\section{Course evaluation}

Thirteen of the 17 workshop participants completed the post-workshop evaluation. All survey respondents either agreed $(15 \%)$ or strongly agreed $(85 \%)$ that the workshop satisfied all course objectives. All survey respondents rated workshop quality as "excellent" and would recommend it to other Internal Medicine residents.

\section{Individual exercises}

All of the exercises received favorable ratings, with "Describe and Draw" and "Personal Responses Tour Part 1 " garnering the highest overall ratings. Full results can be found in Table 3.

\section{Resident commentary}

When asked what they gained from the workshop, multiple residents commented on the power of being intentional and on the ability of art to facilitate encounters with challenging emotions:

"There is value in slowing down and being deliberate in analysis and reflection."

"[I learned] to be cognizant that when I'm 'thinking quickly' and jumping to conclusions, I am at risk of missing important details. In complex situations, it can be helpful to take a more systematic approach."

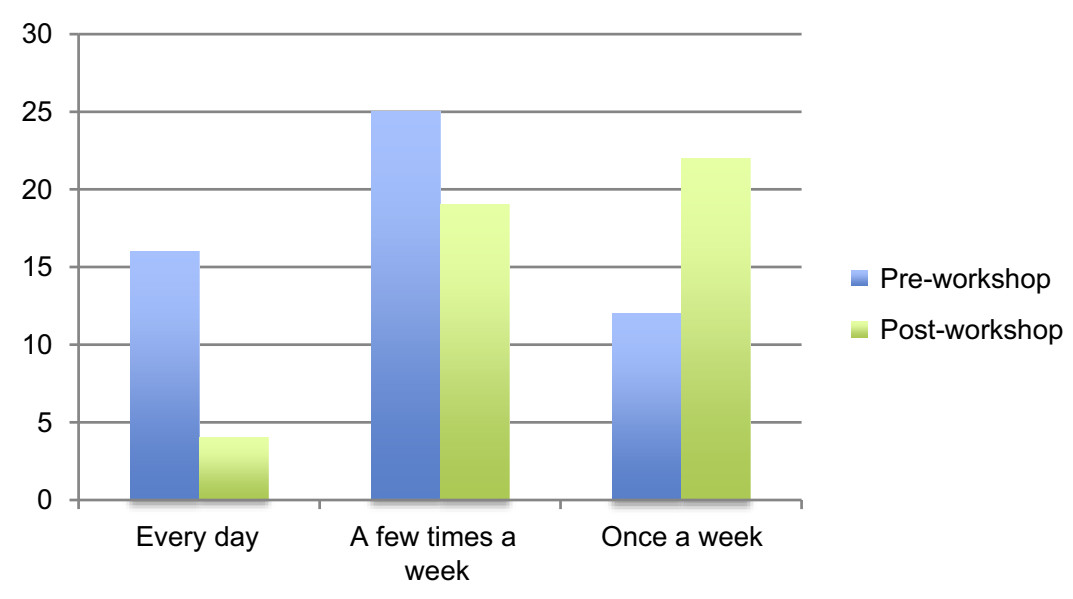

Figure 2 Pre and post-workshop frequency of experiencing depersonalization symptoms.

Table 3 Evaluation of individual workshop exercises (see Supplementary Material for details on the exercises)

\begin{tabular}{|c|c|c|c|c|c|}
\hline & Poor & Fair & Good & Very good & Excellent \\
\hline Elaboration game & 0 & 0 & 0 & 5 (38.5\%) & 8 (6I.5\%) \\
\hline Describe and draw & 0 & I (7.7\%) & 0 & 0 & $12(92.3 \%)$ \\
\hline Step inside & 0 & I (7.7\%) & 5 (38.5\%) & $2(15.4 \%)$ & $5(38.5 \%)$ \\
\hline Personal responses tour part I & 0 & 0 & 0 & $2(15.4 \%)$ & I I (84.6\%) \\
\hline Personal responses tour part 2 & 0 & 0 & $2(15.4 \%)$ & $3(23.1 \%)$ & $8(61.5 \%)$ \\
\hline
\end{tabular}


"Using art to process my emotions helped to express my thoughts and emotions in a way that words couldn't."

"[I learned] the ability to think creatively about ways to cope with the harsh realities of residency."

When asked about the strengths of the workshop, residents noted its novelty and communal nature:

"Interactive, fun, educational, and a brand-new experience."

"Personal response tours were excellent and provided a new tool for how to view art through a specific prompt's lens."

"Gaining the different perspectives of our peers."

"The opportunity to have thoughts/recollections spurred and shared among colleagues."

Regarding areas of improvement, participants commented that they would prefer smaller groups and that the "Step Inside" activity felt redundant.

\section{Discussion}

\section{Medical humanities}

As a proof of concept intervention, the FRAME workshop met its stated objectives and was received positively by participants. Its successful launch demonstrates the feasibility of a single, structured session outside of the hospital environment to promote introspection and community through visual artistic analysis. The favorable course evaluation results encourage continued exploration and repetition with larger cohorts. In future iterations, we recommend eliminating the "Step Inside" activity to shorten the workshop length to three hours, dividing the large group into smaller groups for the entire workshop, incorporating a brief facilitator training session prior to the workshop, and exclusively utilizing the single-item burnout questionnaires to increase survey response rate.

As an investigation into the utility of standardized, introductory artistic analysis in combating burnout, the FRAME workshop delivered promising results. Our findings are compatible with the literature, which suggests that visual artistic analysis can foster tolerance of ambiguity and offer a sanctuary for connection with peers. ${ }^{27}$ Further, they represent, to our knowledge, the first use of the visual arts in burnout analysis. In our cohort, we detected moderate improvements in mean scores for both emotional exhaustion and depersonalization at three months after the workshop. These changes represent roughly twenty percent reductions per burnout category. Finally, although these results are preliminary in nature, we observed decreased frequency of emotional exhaustion and depersonalization symptoms after the workshop, with a trend from experiencing burnout symptoms daily or "a few times a week" symptoms towards experiencing them only "once a week" or less.

Although we observed these heartening results, we recognize the many variables in the lives of participants that will have exerted greater influence on burnout scores than a single 4-hour workshop. Nevertheless, the findings excite and challenge us to repeat the session in future years with larger groups and extended analysis to identify its true benefit. Mechanistically, we propose several means by which use of these exercises may have contributed to increased resilience:

1. Diverse thinking routines increase participants' perspective-taking ability and enhance their ability to find meaning in their work. These skills ideally carry forth into residents' daily lives.

2. The positive reinforcement of these thinking routines creates a safe space to express vulnerability and validates the importance of sharing these emotions.

3. The workshop itself fosters a protective sense of kinship by bringing colleagues together outside of the workplace. The use of artwork as a surrogate for expressing difficult emotions then facilitates honest discussion and provides a formal structure for venting.

\section{Limitations}

One of the limitations facing this study is the lack of a true control cohort for three month analysis, which precludes definitive interpretation of this study's positive results. Additionally, the study is limited by the low response rate $(53 \%)$ to the post-workshop burnout inventory. It is possible that these respondents represent a skewed distribution of participants who rated the workshop favorably or were less burned out.

On a larger scale, the study also faces limitations in terms of the workshop's ultimate role, its sustainability, and its confounding variables. We recognize the importance of "system-based" interventions to target the heart of the burnout epidemic (excessive workload, limited autonomy, inefficient electronic health records, etc.). We offer the FRAME workshop as a creative supplement to the preexisting array of "individual" wellness interventions, intended for use in tandem with continued reform within the healthcare system. Within the realm of individual interventions, we also recognize the limited sustainability of a single, half-day workshop. Ideally, we hope that this 
workshop and others like it can be supported by additional curricular activities such as noon conferences, morning reports, small group sessions, narrative writing courses, and sponsored museum visits, for example. Finally, as noted above, multiple other variables in the lives of residents make it impossible to attribute the positive burnout findings in the FRAME cohort to the workshop exclusively.

\section{Conclusion}

The FRAME workshop was perceived by Internal Medicine residents as a refreshing change of pace. We observed moderate improvements in emotional exhaustion and depersonalization scores following the workshop; however, we do note the low survey response rate and lack of a true control cohort as barriers to definitive interpretation. Although it is not the first workshop focused on visual arts, the standardized use of Artful Thinking Routines ${ }^{17}$ in burnout analysis represents a novel structure that is generalizable and reproducible. The promotion of reflective skills, creative expression, and community building likely contributed to the favorable results noted in this observational study. However, further studies and followup workshops are needed to determine the true effect of the medical humanities on burnout and its sustainability. With that said, we are encouraged to continue seeking ways to infuse meaning back into the daily lives of medical residents and determine the optimal role for this visual artistic workshop in the broader landscape of emerging wellness curricula.

\section{Abbreviations list}

FRAME, Fostering Resilience through Art in Medical Education; IRB, institution review board.

\section{Acknowledgments}

The authors thank the Perelman School of Medicine at the University of Pennsylvania and the Hospital of the University of Pennsylvania Internal Medicine Residency Program for study funding, as well as the Philadelphia Museum of Art for hosting.

The authors report no external funding source for this study.

\section{Disclosure}

The authors declare no conflicts of interest in this work.

\section{References}

1. Ripp JA, Privitera MR, West CP, et al. Well-being in graduate medical education: a call for action. Acad Med. 2017;92 (7):914-917. doi:10.1097/ACM.0000000000001735

2. Moss M, Good VS, Gozal D, Kleinpell R, Sessler CN. An official critical care societies collaborative statement: burnout syndrome in critical care health care professionals: a call for action. Am J Crit Care. 2016;25(4):368-376. doi:10.4037/ajcc2016133

3. Shanafelt TD, Hasan O, Dyrbye LN, et al. Changes in burnout and satisfaction with work-life balance in physicians and the general US working population between 2011 and 2014. Mayo Clin Proc. 2015;90(12):1600-1613. doi:10.1016/j.mayocp.2014.12.011

4. West CP, Shanafelt TD, Kolars JC. Quality of life, burnout, educational debt, and medical knowledge among internal medicine residents. JAMA. 2011;306(9):952-960. doi:10.1001/jama.2011.1247

5. Dyrbye L, Shanafelt T. A narrative review on burnout experienced by medical students and residents. Med Educ. 2016;50(1):132-149. doi:10.1111/medu.2016.50.issue-1

6. Dyrbye LN, West CP, Shanafelt TD. Physician burnout: contributors, consequences, and solutions. J Intern Med. 2018. doi:10.1111/ joim. 12752

7. Williams D, Tricomi G, Gupta J, Janise A. Efficacy of burnout interventions in the medical education pipeline. Acad Psychiatry. 2015;39:47-54. doi:10.1007/s40596-014-0197-5

8. West CP, Dyrbye LN, Erwin PJ, Shanafelt TD. Interventions to prevent and reduce physician burnout: a systematic review and meta-analysis. Lancet. 2016;388(10057):2272-2281. doi:10.1016/ S0140-6736(16)31279-X

9. Gaufberg E, Williams R. Reflection in a museum setting: the personal response tour. J Grad Med Educ. 2011;3(4):546-549. doi:10.4300/ JGME-D-11-00036.1

10. Perry M, Maffulli N, Wilson S, Morrissey D. The effectiveness of arts-based interventions in medical education: a literature review. Med Educ. 2011;45(2):141-148. doi:10.1111/med.2010.45.issue-2

11. Hojat M, Axelrod D, Spandorfer J, Mangione S. Enhancing and sustaining empathy in medical students. Med Teach. 2013;35 (12):996-1001. doi:10.3109/0142159X.2013.802300

12. Zazulak J, Halgren C, Tan M, Grierson LEM. The impact of an arts-based programme on the affective and cognitive components of empathic development. Med Humanit. 2015;41(1):69-74. doi:10.1136/medhum-2014-010584

13. Sands SA, Stanley P, Charon R. Pediatric narrative oncology: interprofessional training to promote empathy, build teams, and prevent burnout. J Support Oncol. 2008;6(7):307-312.

14. Prins JT, Gazendam-Donofrio SM, Dillingh GS, et al. The relationship between reciprocity and burnout in Dutch medical residents. Med Educ. 2008;42(7):721-728. doi:10.1111/j.1365-2923.2008.03041.x

15. Shapiro G, Morrison EH, Boker J. Teaching empathy to first year medical students: evaluation of an elective literature and medicine course. Edu Health. 2004;7(1):73-84. doi:10.1080/135762803100016 56196

16. Mealer M, Conrad D, Evans J, et al. Feasibility and acceptability of a resilience training program for intensive care unit nurses. Am J Crit Care. 2014;23(6):e97-105. doi:10.4037/ajcc2014617

17. President and Fellows of Harvard College; on behalf of Tishman S, Palmer P. Harvard Graduate School of Education. Artful Thinking: Stronger Thinking and Learning through the Power of Art; Final Report; 2006 November. Available from: http://www.pz.harvard. edu/sites/default/files/ArtfulThinkingFinalReport-1.pdf. Accessed 12 May, 2019

18. Housen A. Validating a measure of aesthetic development for museums and schools. ILVS Rev. 1992;2:1-19.

19. Reilly JM, Ring J, Duke L. Visual thinking strategies: a new role for art in medical education. Fam Med. 2005;37(4):250-252. 
20. Yang KT, Yang JH. A study of the effect of a visual arts-based curriculum on the Jefferson scale for physician empathy. BMC Med Educ. 2013;13:142. doi:10.1186/1472-6920-13-99

21. Klugman CM, Peel J, Beckmann-Mendez D. Art rounds: teaching interprofessional students visual thinking strategies at one school. Acad Med. 2011;86:1266-1271. doi:10.1097/ACM.0b013e31822c1427

22. Haidet P, Jarecke J, Adams N, et al. A guiding framework to maximize the power of the arts in medical education: a systematic review and metasynthesis. Med Educ. 2016;50(3):320-331.

23. Maslach C, Jackson SE, Leiter MP. Maslach Burnout Inventory Manual. 3rd ed. Palo Alto, CA: Consulting Psychologists Press; 1996.

24. West CP, Dyrbye LN, Satele DV, et al. Concurrent validity of single-item measures of emotional exhaustion and depersonalization in burnout assessment. J Gen Intern Med. 2012;27(11):1445-1452. doi:10.1007/ s11606-011-1956-6
25. McManus IC, Keeling A, Paice E. Stress, burnout, and doctors' attitudes to work are determined by personality and learning style: a twelve year longitudinal study of UK medical graduates. BMC Med. 2004;2:29. doi:10.1186/1741-7015-2-29

26. Rosenstein L, Torre M, Ramos MA, et al. Prevalence of burnout among physicians: a systematic review. JAMA. 2018;320 (11):1131-1150. doi:10.1001/jama.2018.12777

27. Gowda D, Dubroff R, Willieme A, et al. Art as sanctuary: a four-year mixed-methods evaluation of a visual art course addressing uncertainty through reflection. Acad Med. 2018;93:S8-S13. doi:10.1097/ ACM.0000000000002019 


\section{Supplementary material}

\section{Workshop exercise description (Reference: Harvard Project Zero Artful Thinking Final Report)'}

\section{Elaboration Game}

Process: For this activity, one trainee identifies a specific section of the selected artwork and describes in detail what he or she sees without offering interpretation. Another trainee elaborates on the first participant's observations by adding more detail. A third person elaborates further, and a fourth adds yet more. The art instructor facilitates the observations and ensures that no interpretation is offered. After four participants have described a section in detail, another trainee identifies a separate section of the artwork and the process begins again. After the artwork has been fully described, the group analyzes the details noted in their observations and creates a logical interpretation of the work. The instructors then explicitly tie the exercise to medical practice by soliciting feedback from the residents on how to apply these lessons in their daily routine.

What kind of thinking does this routine encourage? As an introduction to visual artistic analysis, this routine encourages trainees to practice careful observation and diligent attention to detail to collaboratively uncover meaning that may not be immediately apparent. It challenges them to develop elaborate, nuanced, and imaginative verbal descriptions, then synthesize the sum of their observations to create a coherent interpretation of the artwork. This activity aims to strengthen resident awareness, promote understanding of the value underlying the seemingly routine, and encourage patience/flexibility of thinking to withhold judgment until seeking out multiple perspectives.

\section{Back-to-Back Describe and Draw}

Process: For this activity, two trainees sit back-to-back. One trainee (the describer) looks at a work of art and describes it to the other trainee (the listener), who cannot see it. While listening to the describer, the listener draws what they imagine the image to look like. The describer cannot look at the listener's drawing until it is finished. Afterward, the pair discuss their experience guided by the following questions: What descriptive words were most helpful? What was challenging? The exercise is then repeated by switching roles and examining a new work of art. Following the exercise, the large group reconvenes to discuss the experience, and the instructors once again explicitly solicit feedback from trainees regarding medical relevance.

What kind of thinking does this routine encourage? During this routine, students practice relational communication skills. The challenge is not only to describe an image in detail but also to organize descriptions logically. The activity aims to foster an appreciation of different communication styles and to promote the development of meaningful relationships among trainees and their colleagues.

\section{Step Inside}

Process: Participants identify a particular character or object from a selected artwork and reflect on that figure's perspective. Three core questions guide trainees in the process of exploring a viewpoint in a given artwork:

1. What can the person or thing perceive?

2. What might the person or thing know about or believe?

3. What might the person or thing care about?

Answers are then shared and the various viewpoints discussed as a small group. Instructors then explicitly tie the themes discussed back to medical practice.

\section{What kind of thinking does this routine encourage?}

This routine helps students to explore diverse perspectives and viewpoints as they try to imagine events, problems, or issues differently as a means to cope with frustration or misunderstanding.

Personal Responses Tour (in 2 parts) developed by Elizabeth Gaufberg, MD, MPH and Ray Williams, MA

Process: Various prompts were transcribed onto cards that were distributed to participants. Each trainee reflected on one prompt for each of the two portions of this activity. The prompts were designed to invite an emotional response that helps participants find a connection between a work of art and some aspect of life. 
1. Before beginning, three safe space ground rules are outlined: whatever is shared within the group must remain confidential; individuals are encouraged to stay in their comfort zone in revealing personal information; and group members should not question or challenge each other's personal responses.

2. Each participant receives one of the prompts listed below.

3. With that prompt, participants are given 15 minutes to explore the gallery and reflect, with the goal of finding a work of art that resonates with their prompt.

4. Once participants have selected an artwork, they are encouraged to reflect about their prompt as it relates to their artwork and write out their thoughts.

5. Participants then act as a tour guide of their own object by reading their prompt and spending a couple of minutes sharing their responses.

6. The group will then repeat the experience in a different space with Part 2.

- Part 1 (countering dehumanization) prompts:

- Find an artwork that speaks to a professional experience you have had with death and dying. Reflect on that experience as it relates to your chosen artwork.

- Find an artwork that speaks to a professional experience you have had with suffering. Reflect on that experience as it relates to your chosen artwork.

- Find an artwork that speaks to a professional experience in which you have felt overwhelmed. Reflect on that experience as it relates to your chosen artwork.
- Part 2 (rediscovering meaning) prompts; for this iteration, there is a take-home element in which participants are asked to text a photo of the artwork with a message to themselves

- Find an artwork that reminds you of why you chose to become a medical professional.

o Find an artwork that reminds you of something you like about your work.

- Find an artwork that reminds you of an idea you want to hold on to about your work.

- Find an artwork that reminds you of a transformative or affirmative experience you have had at work.

\section{What kind of thinking does this routine encourage?}

This intervention is specifically designed to promote individual reflection, foster empathy, increase appreciation for the psychosocial context of patient experience, and create a safe haven for learners to deepen their relationships with one another.

\section{Reference}

1. President and Fellows of Harvard College; on behalf of Tishman S, Palmer P. Harvard Graduate School of Education. Artful Thinking: Stronger thinking and learning through the power of art; Final Report; November 2006. Available from: http://www.pz.harvard.edu/sites/default/files/ ArtfulThinkingFinalReport-1.pdf. Accessed 12 May, 2019. reviewed, open access journal that aims to present and publish research on Medical Education covering medical, dental, nursing and allied health care professional education. The journal covers undergraduate education, postgraduate training and continuing medical education

including emerging trends and innovative models linking education, research, and health care services. The manuscript management system is completely online and includes a very quick and fair peer-review system. Visit http://www.dovepress.com/testimonials.php to read real quotes from published authors. 